\title{
Dimension Formulae for the Polynomial Algebra as a Module over the Steenrod Algebra in Degrees Less than or Equal to 12
}

\author{
Mbakiso Fix Mothebe ${ }^{1}$, Professor Kaelo ${ }^{2}$ \& Orebonye Ramatebele ${ }^{3}$ \\ 1,2,3 Department of Mathematics, University of Botswana, Pvt Bag 00704, Gaborone, Botswana \\ Correspondence: Mbakiso Fix Mothebe, Department of Mathematics, University of Botswana, Pvt Bag 00704, Gaborone, \\ Botswana. E-mail: mothebemf@mopipi.ub.bw
}

Received: August 10, 2016 Accepted: September 8, 2016 Online Published: September 27, 2016

doi:10.5539/jmr.v8n5p92 URL: http://dx.doi.org/10.5539/jmr.v8n5p92

\begin{abstract}
Let $\mathbf{P}(n)=\mathbb{F}_{2}\left[x_{1}, \ldots, x_{n}\right]$ be the polynomial algebra in $n$ variables $x_{i}$, of degree one, over the field $\mathbb{F}_{2}$ of two elements. The mod-2 Steenrod algebra $\mathcal{A}$ acts on $\mathbf{P}(n)$ according to well known rules. A major problem in algebraic topology is that of determining $\mathcal{A}^{+} \mathbf{P}(n)$, the image of the action of the positively graded part of $\mathcal{A}$. We are interested in the related problem of determining a basis for the quotient vector space $\mathbf{Q}(n)=\mathbf{P}(n) / \mathcal{A}^{+} \mathbf{P}(\mathbf{n})$. Both $\mathbf{P}(n)=\bigoplus_{d \geq 0} \mathbf{P}^{d}(n)$ and $\mathbf{Q}(n)$ are graded, where $\mathbf{P}^{d}(n)$ denotes the set of homogeneous polynomials of degree $d$. In this paper we give explicit formulae for the dimension of $\mathbf{Q}(n)$ in degrees less than or equal to 12 .
\end{abstract}

Keywords: Steenrod squares, polynomial algebra, hit problem.

\section{Introduction}

For $n \geq 1$, let $\mathbf{P}(n)$ be the mod- 2 cohomology group of the $n$-fold product of $\mathbb{R} P^{\infty}$ with itself. Then $\mathbf{P}(n)$ is the polynomial algebra

$$
\mathbf{P}(n)=\mathbb{F}_{2}\left[x_{1}, \ldots, x_{n}\right]
$$

in $n$ variables $x_{i}$, each of degree 1 , over the field $\mathbb{F}_{2}$ of two elements. The mod-2 Steenrod algebra $\mathcal{A}$ is the graded associative algebra generated over $\mathbb{F}_{2}$ by symbols $S q^{i}$ for $i \geq 0$, called Steenrod squares subject to the Adem relations (Adem 1957) and $S q^{0}=1$. Let $\mathbf{P}^{d}(n)$ denote the homogeneous polynomials of degree $d$. The action of the Steenrod squares $S q^{i}: \mathbf{P}^{d}(n) \rightarrow \mathbf{P}^{d+i}(n)$ is determined by the formula:

$$
S q^{i}(u)= \begin{cases}u, & i=0 \\ u^{2}, & \operatorname{deg}(u)=i \\ 0, & \operatorname{deg}(u)<i\end{cases}
$$

and the Cartan formula

$$
S q^{i}(u v)=\sum_{r=0}^{i} S q^{r}(u) S q^{i-r}(v) .
$$

A polynomial $u \in \mathbf{P}^{d}(n)$ is said to be hit if it is in the image of the action of $\mathcal{A}$ on $\mathbf{P}(n)$, that is, if

$$
u=\sum_{i>0} S q^{i}\left(u_{i}\right)
$$

for some $u_{i} \in \mathbf{P}(n)$ of degree $d-i$. Let $\mathcal{A}^{+} \mathbf{P}(n)$ denote the subspace of all hit polynomials. The problem of determining $\mathcal{A}^{+} \mathbf{P}(n)$ is called the hit problem and has been studied by several authors, (Silverman, 1998; Singer, 1991) and (Wood, 1989). We are interested in the related problem of determining a basis for the quotient vector space

$$
\mathbf{Q}(n)=\mathbf{P}(n) / \mathcal{A}^{+} \mathbf{P}(n)
$$

which has also been studied by several authors, (Kameko, 1990; 2003; Peterson, 1987) and (Sum, 2007). Some of the motivation for studying these problems is mentioned in (Nam, 2004). It stems from the Peterson conjecture proved in (Wood, 1989) and various other sources (Peterson, 1989; Singer, 1989).

The following result is useful for determining $\mathcal{A}$-generators for $\mathbf{P}(n)$. Let $\alpha(m)$ denote the number of digits 1 in the binary expansion of $m$.

In [(Wood, 1989) Theorem 1], R.M.W. Wood proved that: 
Theorem 1 (Wood, 1989). Let $u \in \mathbf{P}(n)$ be a monomial of degree d. If $\alpha(n+d)>n$, then $u$ is hit.

Thus $\mathbf{Q}^{d}(n)$ is zero unless $\alpha(n+d) \leq n$ or, equivalently, unless $d$ can be written in the form, $d=\sum_{i=1}^{n}\left(2^{\lambda_{i}}-1\right)$ where $\lambda_{i} \geq 0$. Thus $\mathbf{Q}^{d}(n) \neq 0$ only if $\mathbf{P}^{d}(n)$ contains monomials $v=x_{1}^{2^{\lambda_{1}}-1} \cdots x_{n}^{2^{\lambda_{n}}-1}$ called spikes. For convenience we shall assume that $\lambda_{1} \geq \lambda_{2} \geq \ldots \geq \lambda_{n} \geq 0$. We, in addition, shall consider a special one when $\lambda_{1} \geq \lambda_{2} \geq \ldots \geq \lambda_{s} \geq 0$ and $\lambda_{j-1}=\lambda_{j}$ only if $j=s$ or $\lambda_{j+1}=0$. In this case $v$ is called a minimal spike.

$\mathbf{Q}(n)$ has been explicitly calculated by Peterson in (Peterson, 1987) for $n=1,2$, by (Kameko, 1990) in his thesis, for $n=3$ and independently by (Kameko, 2003) and (Sum, 2007) for $n=4$. In this work we shall, unless otherwise stated, be concerned with a basis for $\mathbf{Q}(n)$ consisting of 'admissible monomials', as defined below. Thus when we write $u \in \mathbf{Q}^{d}(n)$ we mean that $u$ is an admissible monomial of degree $d$.

We define what it means for a monomial $b=x_{1}^{e_{1}} \cdots x_{n}^{e_{n}} \in \mathbf{P}(n)$ to be admissible. Write $e_{i}=\sum_{j \geq 0} \alpha_{j}\left(e_{i}\right) 2^{j}$ for the binary expansion of each exponent $e_{i}$. The expansions are then assembled into a matrix $\beta(b)=\left(\alpha_{j}\left(e_{i}\right)\right)$ of digits 0 or 1 with $\alpha_{j}\left(e_{i}\right)$ in the $(i, j)$-th position of the matrix.

We then associate with $b$, two sequences,

$$
\begin{aligned}
w(b) & =\left(w_{0}(b), w_{1}(b), \ldots, w_{j}(b), \ldots\right), \\
e(b) & =\left(e_{1}, e_{2}, \ldots, e_{n}\right)
\end{aligned}
$$

where $w_{j}(b)=\sum_{i=1}^{n} \alpha_{j}\left(e_{i}\right)$ for each $j \geq 0 . w(b)$ is called the weight vector of the monomial $b$ and $e(b)$ is called the exponent vector of the monomial $b$.

Given two sequences $p=\left(u_{0}, u_{1}, \ldots, u_{l}, 0, \ldots\right), q=\left(v_{0}, v_{1}, \ldots, v_{l}, 0, \ldots\right)$, we say $p<q$ if there is a positive integer $k$ such that $u_{i}=v_{i}$ for all $i<k$ and $u_{k}<v_{k}$. We are now in a position to define an order relation on monomials.

Definition 1. Let $a, b$ be monomials in $\mathbf{P}(n)$. We say that $a<b$ if one of the following holds:

1. $w(a)<w(b)$,

2. $w(a)=w(b)$ and $e(a)<e(b)$.

Note that the order relation on the set of sequences is the lexicographical one.

Following (Kameko, 1990) we define:

Definition 2. A monomial $b \in \mathbf{P}(n)$ is said to be inadmissible if there exist monomials $b_{1}, b_{2}, \ldots, b_{r} \in \mathbf{P}(n)$ with $b_{j}<b$ for each $j, 1 \leq j \leq r$, such that

$$
b \equiv\left(\sum_{j=1}^{r} b_{j}\right) \bmod \mathcal{A}^{+} \mathbf{P}(n) .
$$

$b$ is said to be admissible if it is not inadmissible.

Clearly the set of all admissible monomials in $\mathbf{P}(n)$ form a basis for $\mathbf{Q}(n)$.

To put our work in context we require some preliminary observations. For each $r, 1 \leq r \leq n$, let

$$
\mathbf{X}(r)=\operatorname{Span}\left\{x_{1}^{m_{1}} \cdots x_{r}^{m_{r}} \in \mathbf{P}(r) \mid m_{1} m_{2} \cdots m_{r} \neq 0\right\} .
$$

Then $\mathbf{X}(r)$ is an $\mathcal{A}$-submodule of $\mathbf{P}(r)$. Let

$$
\mathbf{W}(r)=\mathbf{X}(r) / \mathcal{A}^{+} \mathbf{X}(r) .
$$

Then for each $n \geq 1$ we have a direct sum decomposition:

$$
\mathbf{Q}(n) \cong \bigoplus_{r=1}^{n} \bigoplus_{k=1}^{\left(\begin{array}{c}
n \\
r
\end{array}\right)} \mathbf{W}(r)
$$

Thus for any integer $d>0$ we have the following inexplicit formula for the dimension of $\mathbf{Q}^{d}(n)$ :

$$
\operatorname{dim}\left(\mathbf{Q}^{d}(n)\right)=\sum_{r=1}^{n}\left(\begin{array}{l}
n \\
r
\end{array}\right) \operatorname{dim}\left(\mathbf{W}^{d}(r)\right) .
$$


But $\mathbf{Q}(n)$ is known for , $1 \leq n \leq 4$, and in some cases when $n=5$. This gives rise to the following explicit formula for $\mathbf{Q}^{d}(n)$ for $d \leq 5$.

$$
\begin{aligned}
& \operatorname{dim}\left(\mathbf{Q}^{1}(n)\right)=\left(\begin{array}{l}
n \\
1
\end{array}\right) . \\
& \operatorname{dim}\left(\mathbf{Q}^{2}(n)\right)=\left(\begin{array}{l}
n \\
2
\end{array}\right) . \\
& \operatorname{dim}\left(\mathbf{Q}^{3}(n)\right)=\left(\begin{array}{l}
n \\
1
\end{array}\right)+\left(\begin{array}{l}
n \\
2
\end{array}\right)+\left(\begin{array}{l}
n \\
3
\end{array}\right) . \\
& \operatorname{dim}\left(\mathbf{Q}^{4}(n)\right)=\left(\begin{array}{l}
n \\
2
\end{array}\right) \cdot 2+\left(\begin{array}{l}
n \\
3
\end{array}\right) \cdot 2+\left(\begin{array}{l}
n \\
4
\end{array}\right) . \\
& \operatorname{dim}\left(\mathbf{Q}^{5}(n)\right)=\left(\begin{array}{l}
n \\
3
\end{array}\right) \cdot 3+\left(\begin{array}{l}
n \\
4
\end{array}\right) \cdot 3+\left(\begin{array}{l}
n \\
5
\end{array}\right) .
\end{aligned}
$$

We follow the convention that $\left(\begin{array}{c}n \\ i\end{array}\right)=0$ if $i>n$.

Our main result is Theorem 2 below which consists of explicit formulae for $\operatorname{dim}\left(\mathbf{Q}^{d}(n)\right), 6 \leq d \leq 12$.

Theorem 2. For all $n \geq 1$ :

$$
\begin{aligned}
& \operatorname{dim}\left(\mathbf{Q}^{6}(n)\right)=\left(\begin{array}{l}
n \\
2
\end{array}\right)+\left(\begin{array}{l}
n \\
3
\end{array}\right) \cdot 3+\left(\begin{array}{l}
n \\
4
\end{array}\right) \cdot 6+\left(\begin{array}{l}
n \\
5
\end{array}\right) \cdot 4+\left(\begin{array}{l}
n \\
6
\end{array}\right) . \\
& \operatorname{dim}\left(\mathbf{Q}^{7}(n)\right)=\left(\begin{array}{l}
n \\
1
\end{array}\right)+\left(\begin{array}{l}
n \\
2
\end{array}\right)+\left(\begin{array}{l}
n \\
3
\end{array}\right) \cdot 4+\left(\begin{array}{l}
n \\
4
\end{array}\right) \cdot 9+\left(\begin{array}{l}
n \\
5
\end{array}\right) \cdot 10+\left(\begin{array}{l}
n \\
6
\end{array}\right) \cdot 5+\left(\begin{array}{l}
n \\
7
\end{array}\right) . \\
& \operatorname{dim}\left(\mathbf{Q}^{8}(n)\right)=\left(\begin{array}{l}
n \\
2
\end{array}\right) \cdot 3+\left(\begin{array}{l}
n \\
3
\end{array}\right) \cdot 6+\left(\begin{array}{l}
n \\
4
\end{array}\right) \cdot 13+\left(\begin{array}{l}
n \\
5
\end{array}\right) \cdot 19+\left(\begin{array}{l}
n \\
6
\end{array}\right) \cdot 15+\left(\begin{array}{l}
n \\
7
\end{array}\right) \cdot 6+\left(\begin{array}{l}
n \\
8
\end{array}\right) . \\
& \operatorname{dim}\left(\mathbf{Q}^{9}(n)\right)=\left(\begin{array}{l}
n \\
3
\end{array}\right) \cdot 7+\left(\begin{array}{l}
n \\
4
\end{array}\right) \cdot 18+\left(\begin{array}{l}
n \\
5
\end{array}\right) \cdot 31+\left(\begin{array}{l}
n \\
6
\end{array}\right) \cdot 34+\left(\begin{array}{l}
n \\
7
\end{array}\right) \cdot 21+\left(\begin{array}{l}
n \\
8
\end{array}\right) \cdot 7+\left(\begin{array}{l}
n \\
9
\end{array}\right) . \\
& \operatorname{dim}\left(\mathbf{Q}^{10}(n)\right)=\left(\begin{array}{l}
n \\
2
\end{array}\right) \cdot 2+\left(\begin{array}{l}
n \\
3
\end{array}\right) \cdot 8+\left(\begin{array}{l}
n \\
4
\end{array}\right) \cdot 26+\left(\begin{array}{l}
n \\
5
\end{array}\right) \cdot 50+\left(\begin{array}{l}
n \\
6
\end{array}\right) \cdot 65+\left(\begin{array}{l}
n \\
7
\end{array}\right) \cdot 55 \\
& +\left(\begin{array}{l}
n \\
8
\end{array}\right) \cdot 28+\left(\begin{array}{l}
n \\
9
\end{array}\right) \cdot 8+\left(\begin{array}{c}
n \\
10
\end{array}\right) \text {. } \\
& \operatorname{dim}\left(\mathbf{Q}^{11}(n)\right)=\left(\begin{array}{l}
n \\
3
\end{array}\right) \cdot 8+\left(\begin{array}{l}
n \\
4
\end{array}\right) \cdot 32+\left(\begin{array}{l}
n \\
5
\end{array}\right) \cdot 75+\left(\begin{array}{l}
n \\
6
\end{array}\right) \cdot 115+\left(\begin{array}{l}
n \\
7
\end{array}\right) \cdot 120+\left(\begin{array}{l}
n \\
8
\end{array}\right) \cdot 83 \\
& +\left(\begin{array}{c}
n \\
9
\end{array}\right) \cdot 36+\left(\begin{array}{c}
n \\
10
\end{array}\right) \cdot 9+\left(\begin{array}{c}
n \\
11
\end{array}\right) \text {. } \\
& \operatorname{dim}\left(\mathbf{Q}^{12}(n)\right)=\left(\begin{array}{l}
n \\
4
\end{array}\right) \cdot 21+\left(\begin{array}{l}
n \\
5
\end{array}\right) \cdot 85+\left(\begin{array}{l}
n \\
6
\end{array}\right) \cdot 176+\left(\begin{array}{l}
n \\
7
\end{array}\right) \cdot 231+\left(\begin{array}{l}
n \\
8
\end{array}\right) \cdot 203 \\
& +\left(\begin{array}{c}
n \\
9
\end{array}\right) \cdot 109+\left(\begin{array}{c}
n \\
10
\end{array}\right) \cdot 45+\left(\begin{array}{c}
n \\
11
\end{array}\right) \cdot 10+\left(\begin{array}{c}
n \\
12
\end{array}\right) \text {. }
\end{aligned}
$$

Our proof of Theorem 2 is deferred until Section 3.

Ignoring the terms for which $\mathbf{W}^{d}(r)$ is trivial, the above formulae yield Table 1 below for the dimension of $\mathbf{Q}^{d}(n), 1 \leq d \leq$ 12. The numbers on the top row represent $n$, the number of variables while the serial numbers in the first column represent $d$, the degree of $\mathbf{Q}(n)$.

Our work is organized as follows. In Section 2, we recall some results on admissible monomials and hit monomials in $\mathbf{P}(n)$. In Section 3 we prove Theorem 2 .

\section{Preliminaries}

In this section we recall some results in (Kameko, 1990; Silverman, 1998; Singer, 1991) and (Mothebe, 2016) on admissible monomials and hit monomials in $\mathbf{P}(n)$.

The following theorem has been used to great effect by Kameko and Sum in computing a basis for $\mathbf{Q}(3)$ and $\mathbf{Q}(4)$ respectively.

Theorem 3 (Kameko, 1990; Sum, 2010). Let $a, b$ be monomials in $\mathbf{P}(n)$ such that $w_{j}(a)=0$ for $j>r>0$. If $b$ is inadmissible, then $a b^{2^{r}}$ is also inadmissible.

Up to permutation of representatives weight order provides a total order relation amongst spikes in a given degree. 
Table 1. Results obtained using Theorem 2

\begin{tabular}{cc|cccccccccccc}
\hline$d$ & $n$ & 1 & 2 & 3 & 4 & 5 & 6 & 7 & 8 & 9 & 10 & 11 & 12 \\
\hline 1 & 1 & & & & & & & & & & & \\
2 & 0 & 1 & & & & & & & & & & \\
3 & 1 & 3 & 7 & & & & & & & & & \\
4 & 0 & 2 & 8 & 21 & & & & & & & & \\
5 & 0 & 0 & 3 & 15 & 46 & & & & & & & \\
6 & 0 & 1 & 6 & 24 & 74 & 190 & & & & & & \\
7 & 1 & 3 & 10 & 35 & 110 & 301 & 729 & & & & & \\
8 & 0 & 3 & 15 & 55 & 174 & 489 & 1238 & 2863 & & & & \\
9 & 0 & 0 & 7 & 46 & 191 & 630 & 1785 & 4515 & 10438 & & & \\
10 & 0 & 2 & 14 & 70 & 280 & 945 & 2792 & 7412 & 18020 & 40701 & & \\
11 & 0 & 0 & 8 & 64 & 315 & 1205 & 3900 & 11151 & 28917 & 69234 & 155035 & \\
12 & 0 & 0 & 0 & 21 & 190 & 1001 & 3983 & 13209 & 38402 & 100880 & 243737 & 550847 \\
\hline
\end{tabular}

It is easy to show that a spike $v=x_{1}^{2^{\lambda_{1}}-1} \cdots x_{n}^{2^{\lambda_{n}}-1} \in \mathbf{P}^{d}(n)$ is a minimal spike if its weight order is minimal with respect to other spikes of degree $d$. In [(Kameko 1990), Theorem 4.2] Masaki Kameko proved that:

Theorem 4 (Kameko, 1990). Let $d$ be a positive integer and let $v$ be the minimal spike of degree $2 d+n$. Define a linear mapping, $f: \mathbf{P}^{d}(n) \rightarrow \mathbf{P}^{2 d+n}(n)$, by,

$$
f\left(x_{1}^{m_{1}} \cdots x_{n}^{m_{n}}\right)=x_{1}^{2 m_{1}+1} \cdots x_{n}^{2 m_{n}+1} .
$$

If $w_{0}(v)=n$, then $f$ induces an isomorphism $f_{*}: \mathbf{Q}^{d}(n) \rightarrow \mathbf{Q}^{2 d+n}(n)$.

From Wood's theorem and the above result of Kameko the problem of determining $\mathcal{A}$-generators for $\mathbf{P}(n)$ is reduced to the cases for which $w_{0}(v) \leq n-1$ whenever $v$ is a minimal spike of a given degree $d$.

We recall the following result of Singer on hit polynomials in $\mathbf{P}(n)$. In [(Singer, 1991), Theorem 1.2] W. M. Singer proved that:

Theorem 5 (Singer, 1991). Let $b \in \mathbf{P}(n)$ be a monomial of degree $d$, where $\alpha(n+d) \leq n$. Let $v$ be the minimal spike of degree $d$. If $w(b)<w(v)$, then $b$ is hit.

We note the following stronger version of Theorem 5. Let $b$ be a monomial of degree $d$. For $l>0$ define $d_{l}(b)$ to be the integer $d_{l}(b)=\sum_{j \geq l} w_{j}(b) 2^{j-l}$.

In [(Silverman, 1998), Theorem 1.2], J. H. Silverman proved that:

Theorem 6 (Silverman, 1998). Let $b \in \mathbf{P}(n)$ be a monomial of degree $d$, where $\alpha(n+d) \leq n$. Let $v$ be the minimal spike of degree $d$. If $d_{l}(b)>d_{l}(v)$ for some $l \geq 1$, then $b$ is hit.

We shall require the following result of (Mothebe, 2016):

Theorem 7 (Mothebe, 2016). If $u=x_{1}^{m_{1}} \cdots x_{k}^{m_{k}} \in \mathbf{P}^{d}(k)$ and $v=x_{1}^{e_{1}} \cdots x_{r}^{e_{r}} \in \mathbf{P}^{d^{\prime}}(r)$ are admissible monomials, then for each permutation $\sigma \in S_{k+r}$ for which $\sigma(i)<\sigma(j), i<j \leq k$ and $\sigma(s)<\sigma(t), k<s<t \leq k+r$, the monomial

$$
x_{\sigma(1)}^{m_{1}} \cdots x_{\sigma(k)}^{m_{k}} x_{\sigma(k+1)}^{e_{1}} \cdots x_{\sigma(k+r)}^{e_{r}} \in \mathbf{P}^{d+d^{\prime}}(k+r)
$$

is admissible.

Theorem 7 is a generalization of the following result of the (Mothebe \& Uys, 2015).

Let $u=x_{1}^{m_{1}} \cdots x_{n-1}^{m_{n-1}} \in \mathbf{P}(n-1)$ be a monomial of degree $d^{\prime}$. Given any pair of integers $(j, \lambda), 1 \leq j \leq n, \lambda \geq 0$, let $h_{j}^{\lambda}(u)$ denote the monomial $x_{1}^{m_{1}} \cdots x_{j-1}^{m_{j-1}} x_{j}^{2^{\lambda}-1} x_{j+1}^{m_{j}} \cdots x_{n}^{m_{n-1}} \in \mathbf{P}^{d^{\prime}+\left(2^{\lambda}-1\right)}(n)$.

Theorem 8 (Mothebe \& Uys, 2015). Let $u \in \mathbf{P}(n-1)$ be a monomial of degree $d^{\prime}$, where $\alpha\left(d^{\prime}+n-1\right) \leq n-1$. If $u$ is admissible, then for each pair of integers $(j, \lambda), 1 \leq j \leq n, \lambda \geq 0, h_{j}^{\lambda}(u)$ is admissible. 


\section{Proof of Theorem 2}

The result of Theorem 2 is a consequence of Lemma 9 and Lemma 10 which we prove below.

Lemma 9. If $a=x_{1}^{m_{1}} \ldots x_{n}^{m_{n}} \in \mathbf{X}(n)$ is an admissible monomial then $m_{1}=2^{\lambda}-1$ for some $\lambda \geq 1$.

Proof. The lemma is clearly true if $n=1$. Suppose that $m_{1}=2^{\lambda}-2$. Let $b=x_{1}^{e_{1}} \ldots x_{n}^{e_{n}}$ be the monomial obtained from $a$ by replacing $m_{1}$ by $2^{\lambda}-3$. Then $a=S q^{1}(b)+x_{1}^{2^{\lambda}-3} S q^{1}\left(x_{2}^{m_{2}} \ldots x_{n}^{m_{n}}\right)$ and the fact that all terms in $x_{1}^{2^{\lambda}-3} S q^{1}\left(x_{2}^{m_{2}} \ldots x_{n}^{m_{n}}\right)$ are of lower order than $a$ shows that $a$ is inadmissible. But every monomial with $m_{1} \neq 2^{\lambda}-1$ is of the form $c d^{2^{r}}$ for some monomial $d=x_{1}^{t_{1}} \ldots x_{n}^{t_{n}}$ with $t_{1}=2^{\lambda}-2$ so the general result follows from Theorem 3 .

Further to Lemma 9, suppose that $a=x_{1}^{2^{\lambda}-1} \ldots x_{n}^{m_{n}}$ with $\lambda \geq 2$. If $m_{2}=2^{k}-2$ for some $k \geq 2$, then $a$ is inadmissible.

Recall that for each $r, 1 \leq r \leq n$,

$$
\mathbf{X}(r)=\operatorname{Span}\left\{x_{1}^{m_{1}} \cdots x_{r}^{m_{r}} \in \mathbf{P}(r) \mid m_{1} m_{2} \cdots m_{r} \neq 0\right\} .
$$

Then $\mathbf{X}(r)$ is an $\mathcal{A}$-submodule of $\mathbf{P}(r)$. Let

$$
\mathbf{W}(r)=\mathbf{X}(r) / \mathcal{A}^{+} \mathbf{X}(r) .
$$

Then for each $n \geq 1$ we have a direct sum decomposition:

$$
\mathbf{Q}(n) \cong \bigoplus_{r=1}^{n} \bigoplus_{k=1}^{\left(\begin{array}{c}
n \\
r
\end{array}\right)} \mathbf{W}(r)
$$

Thus for any integer $d>0$ we have the following inexplicit formula for the dimension of $\mathbf{Q}^{d}(n)$

$$
\operatorname{dim}\left(\mathbf{Q}^{d}(n)\right)=\sum_{r=1}^{n}\left(\begin{array}{l}
n \\
r
\end{array}\right) \operatorname{dim}\left(\mathbf{W}^{d}(r)\right) .
$$

The following lemma evaluates Formula (2) explicitly in some cases. It gives explicit formulae for $\operatorname{dim}\left(\mathbf{W}^{d}(n)\right)$ for those cases that enables us to obtain $\operatorname{dim}\left(\mathbf{Q}^{d}(n)\right)$ for all values of $n$ in the range, $1 \leq n \leq 12$.

\section{Lemma 10.}

$$
\begin{aligned}
& \operatorname{dim}\left(\mathbf{W}^{n}(n)\right) \quad=1 \\
& \operatorname{dim}\left(\mathbf{W}^{n}(n-1)\right)=n-2 \\
& \operatorname{dim}\left(\mathbf{W}^{n}(n-2)\right)=\left(\begin{array}{c}
n-2 \\
2
\end{array}\right) \\
& \operatorname{dim}\left(\mathbf{W}^{n}(n-3)\right)=\left(\begin{array}{c}
n-4 \\
3
\end{array}\right)+(n-3)(n-5) \\
& \operatorname{dim}\left(\mathbf{W}^{n}(n-4)\right)=\left(\left(\begin{array}{c}
n-5 \\
4
\end{array}\right)-1\right)+\left(\begin{array}{c}
n-4 \\
2
\end{array}\right)+(n-4) \cdot\left(\begin{array}{c}
n-6 \\
2
\end{array}\right)+\left(\begin{array}{c}
n-5 \\
2
\end{array}\right) \\
& \operatorname{dim}\left(\mathbf{W}^{n}(n-5)\right)=n-6+\frac{(n-5) !}{2 \cdot(n-7)(n-9) !}+\left(\begin{array}{c}
n-5 \\
2
\end{array}\right)+\left(\begin{array}{c}
n-6 \\
2
\end{array}\right)+(n-5) \cdot\left(\begin{array}{c}
n-7 \\
3
\end{array}\right) \\
& +2 \cdot\left(\sum_{i=2}^{n-8}\left(\begin{array}{l}
i \\
2
\end{array}\right)\right)+\left(\begin{array}{c}
n-8 \\
2
\end{array}\right)+\left(\left(\begin{array}{c}
n-7 \\
2
\end{array}\right)-1\right) \\
& 10 \leq n \leq 12 \\
& \operatorname{dim}\left(\mathbf{W}^{n}(n-6)\right)=n-6+((n-7)(n-8)-2)+\left(\begin{array}{c}
n-6 \\
2
\end{array}\right)+\left(\begin{array}{c}
n-6 \\
3
\end{array}\right) \\
& +(n-6) \cdot\left(\begin{array}{c}
n-8 \\
2
\end{array}\right)+\frac{(n-7) !}{4 \cdot(n-11) !}+(n-8) \cdot\left(\begin{array}{c}
n-9 \\
2
\end{array}\right)+\left(\begin{array}{c}
n-9 \\
2
\end{array}\right) \\
& +\left(\left(\begin{array}{c}
n-6 \\
3
\end{array}\right)-1\right)+2 \cdot\left(\begin{array}{c}
n-7 \\
3
\end{array}\right)-(n-9)
\end{aligned}
$$

Proof. For $n \geq 1$ the basis of $\mathbf{X}^{n}(n)$ consists of the monomial $x_{1} x_{2} \cdots x_{n-1} x_{n}$. For $n \geq 3$ the basis of $\mathbf{X}^{n}(n-1)$ consists of the monomial $x_{1} x_{2} \cdots x_{n-2} x_{n-1}^{2}$ and its permutation representatives. For $n \geq 6$ the basis of $\mathbf{X}^{n}(n-2)$ consists of the monomials:

$$
\left\{a_{n-2}=x_{1} x_{2} \cdots x_{n-4} x_{n-3}^{2} x_{n-2}^{2}, b_{n-2}=x_{1} x_{2} \cdots x_{n-4} x_{n-3} x_{n-2}^{3}\right\}
$$

and their permutation representatives. For convenience we shall ignore those monomials in $\mathbf{X}^{n}(n-i)$ which, by Theorem 6 , are hit. For $n \geq 7$ the basis of $\mathbf{X}^{n}(n-3)$ consists of the monomials:

$$
\left\{a_{n-3}=x_{1} x_{2} \cdots x_{n-6} x_{n-5}^{2} x_{n-4}^{2} x_{n-3}^{2}, b_{n-3}=x_{1} x_{2} \cdots x_{n-5} x_{n-4}^{2} x_{n-3}^{3}, c_{n-3}=x_{1} x_{2} \cdots x_{n-4} x_{n-3}^{4}\right\}
$$


and their permutation representatives. If $n \geq 8$, the basis of $\mathbf{X}^{n}(n-4)$ consists of the monomials:

$$
\left\{a_{n-4}=x_{1} \cdots x_{n-7} x_{n-6}^{2} x_{n-5}^{2} x_{n-4}^{3}, b_{n-4}=x_{1} \cdots x_{n-6} x_{n-5}^{2} x_{n-4}^{4}, c_{n-4}=x_{1} \cdots x_{n-6} x_{n-5}^{3} x_{n-4}^{3}, e_{n-4}=x_{1} \cdots x_{n-6} x_{n-5} x_{n-4}^{5}\right\}
$$

and their permutation representatives, while

$$
d_{n-4}=x_{1} x_{2} \cdots x_{n-8} x_{n-7}^{2} x_{n-6}^{2} x_{n-5}^{2} x_{n-4}^{2}
$$

and its permutation representatives have to be included in the list when $n \geq 10$.

If $n \geq 9$ the basis of $\mathbf{X}^{n}(n-5)$ consists of the monomials:

$$
\left\{a_{n-5}=x_{1} \cdots x_{n-8} x_{n-7}^{2} x_{n-6}^{3} x_{n-5}^{3}, b_{n-5}=x_{1} x_{2} \cdots x_{n-7} x_{n-6}^{3} x_{n-5}^{4}, c_{n-5}=x_{1} x_{2} \cdots x_{n-7} x_{n-6}^{2} x_{n-5}^{5}, d_{n-5}=x_{1} x_{2} \cdots x_{n-6} x_{n-5}^{6}\right\}
$$

and their permutation representatives, while

$$
e_{n-5}=x_{1} x_{2} \cdots x_{n-9} x_{n-8}^{2} x_{n-7}^{2} x_{n-6}^{2} x_{n-5}^{3}, f_{n-5}=x_{1} x_{2} \cdots x_{n-8} x_{n-7}^{2} x_{n-6}^{2} x_{n-5}^{4}
$$

and their permutation representatives have to be added to the list when $n \geq 10$ and

$$
g_{n-5}=x_{1} x_{2} \cdots x_{n-11} x_{n-10} x_{n-9}^{2} x_{n-8}^{2} x_{n-7}^{2} x_{n-6}^{2} x_{n-5}^{2}
$$

and its permutation representatives have to be added to the list when $n \geq 13$.

If $n \geq 11$ the basis of $\mathbf{X}^{n}(n-6)$ consists of the monomials:

$$
\begin{aligned}
& \left\{a_{n-6}=x_{1} \cdots x_{n-9} x_{n-8}^{3} x_{n-7}^{3} x_{n-6}^{3}, b_{n-6}=x_{1} \cdots x_{n-7} x_{n-6}^{7}, c_{n-6}=x_{1} \cdots x_{n-8} x_{n-7}^{3} x_{n-6}^{5}, d_{n-6}=x_{1} \cdots x_{n-10} x_{n-9}^{2} x_{n-8}^{2} x_{n-7}^{3} x_{n-6}^{3},\right. \\
& \left.e_{n-6}=x_{1} x_{2} \cdots x_{n-9} x_{n-8}^{2} x_{n-7}^{3} x_{n-6}^{4}, f_{n-6}=x_{1} x_{2} \cdots x_{n-8} x_{n-7}^{2} x_{n-6}^{6}, g_{n-6}=x_{1} x_{2} \cdots x_{n-9} x_{n-8}^{2} x_{n-7}^{2} x_{n-6}^{5}\right\}
\end{aligned}
$$

and their permutation representatives, while

$$
h_{n-6}=x_{1} x_{2} \cdots x_{n-11} x_{n-10}^{2} x_{n-9}^{2} x_{n-8}^{2} x_{n-7}^{2} x_{n-6}^{3}, k_{n-6}=x_{1} x_{2} \cdots x_{n-10} x_{n-9}^{2} x_{n-8}^{2} x_{n-7}^{2} x_{n-6}^{4}
$$

and their permutation representatives have to be added to the list when $n \geq 13$ and

$$
l_{n-6}=x_{1} x_{2} \cdots x_{n-12} x_{n-11}^{2} x_{n-10}^{2} x_{n-9}^{2} x_{n-8}^{2} x_{n-7}^{2} x_{n-6}^{2}
$$

and its permutation representatives has to be added to the list when $n \geq 14$.

We claim that for all pairs $(n, i), 1 \leq i \leq 4$, and all $n$ as specified in Lemma $10, \mathbf{W}^{n+1}(n-i+1)$ is the vector space sum

$$
\mathbf{W}^{n+1}(n-i+1)=\sum_{j=1}^{n-i+1} h_{j}^{1}\left(\mathbf{W}^{n}(n-i)\right),
$$

while this is true for $n=10,11$ when $i=5$ and for $n=11$ when $i=6$. This shall suffice for a proof of Lemma 10 since the formulae in the lemma give the number of elements $\mathbf{W}^{n+1}(n-i+1)$ obtained from $\mathbf{W}^{n}(n-i)$ in this inductive manner.

For each $i, 0 \leq i \leq 6$, we identify, for the initial value $n_{0}$ of $n$, all elements in $\mathbf{X}^{n_{0}}\left(n_{0}-i\right)$ which are admissible, that is, we determine the admissible monomial basis for $\mathbf{W}^{n_{0}}\left(n_{0}-i\right)$. We then show, for a given integer $i$, and all $n \geq n_{0}$, that each element of $\mathbf{X}^{n+1}(n-i+1)$ which does not belong to the set

$$
\left\{h_{j}^{1}\left(x_{1}^{m_{1}} \cdots x_{n-i}^{m_{n-i}}\right) \mid x_{1}^{m_{1}} \cdots x_{n-i}^{m_{n-i}} \in \mathbf{W}^{n}(n-i), 1 \leq j \leq n-i+1\right\},
$$

is inadmissible.

Clearly $\operatorname{dim}\left(\mathbf{W}^{n}(n)\right)=1$ for all $n \geq 1$ since $x_{1} x_{2} \cdots x_{n-1} x_{n}$ is the only admissible element in $\mathbf{X}^{n}(n)$, while $\operatorname{dim}\left(\mathbf{W}^{n}(n-1)\right)=n-2$ for all $n \geq 3$ since there are $n-2$ permutation representatives of $x_{1} x_{2} \cdots x_{n-2} x_{n-1}^{2}$ which are admissible.

If $i=2$, then $n_{0}=6$ and it is known that $\mathbf{W}^{6}(4)$ is generated by $x_{1} x_{2} x_{3} x_{4}^{3}$ and all its permutation representatives together with the monomials $x_{1} x_{2}^{2} x_{3} x_{4}^{2}$ and $x_{1} x_{2} x_{3}^{2} x_{4}^{2}$. If $n>6$, then all permutation representatives of $b_{n-2}$ are admissible and the only permutation representatives of $a_{n-2}$ in $\mathbf{X}^{n}(n-2)$ that may not be obtained from the basis of $\mathbf{W}^{6}(4)$ by inductively applying Equation (3) are those of the form $x_{1}^{2} \cdots x_{n-2}^{m_{n-2}}$ as well as the monomial $x_{1} x_{2}^{2} x_{3}^{2} x_{4} \cdots x_{n-2}$, all of which are clearly 
inadmissible. Thus $\operatorname{dim}\left(\mathbf{W}^{n}(n-2)\right)=\left(\begin{array}{c}n-2 \\ 2\end{array}\right)$, for all $n \geq 6$, since $a_{n-2}, b_{n-2}$ have, respectively, $\left(\begin{array}{c}n-3 \\ 2\end{array}\right)-1, n-2$ permutation representatives which are admissible and $\left(\begin{array}{c}n-3 \\ 2\end{array}\right)-1+n-2=\left(\begin{array}{c}n-2 \\ 2\end{array}\right)$.

If $i=3$, then $n_{0}=7$ and it is known that $\mathbf{W}^{7}(4)$ is generated by the monomials

$$
x_{1} x_{2}^{2} x_{3}^{2} x_{4}^{2} \text { and } x_{1} x_{2} x_{3}^{2} x_{4}^{3}, x_{1} x_{2} x_{3}^{3} x_{4}^{2}, x_{1} x_{2}^{3} x_{3} x_{4}^{2}, x_{1}^{3} x_{2} x_{3} x_{4}^{2}, x_{1} x_{2}^{2} x_{3} x_{4}^{3} x_{1} x_{2}^{2} x_{3}^{3} x_{4}, x_{1} x_{2}^{3} x_{3}^{2} x_{4}, x_{1}^{3} x_{2} x_{3}^{2} x_{4} .
$$

It is easy to show that the monomial $c_{n-3}$ and all its permutation representatives are inadmissible for all $n \geq 7$.

If $n>7$, then the only permutation representatives of $a_{n-3}$ and $b_{n-3}$ in $\mathbf{X}^{n}(n-3)$ that may not be obtained from the basis of $\mathbf{W}^{7}(4)$ by inductively applying Equation (3) are those of the form $x_{1}^{2} \cdots x_{n-3}^{m_{n-3}}$ as well as the monomial $x_{1}^{3} x_{2}^{2} x_{3} x_{4} \cdots x_{n-3}$, all of which are clearly inadmissible. Thus $\operatorname{dim}\left(\mathbf{W}^{n}(n-3)\right)=\left(\begin{array}{c}n-4 \\ 3\end{array}\right)+(n-3)(n-5)$, for all $n \geq 7$, since $a_{n-3}, b_{n-3}$ have, respectively, $\left(\begin{array}{c}n-4 \\ 3\end{array}\right),(n-3)(n-5)$ permutation representatives which are admissible.

If $i=4$, then $n_{0}=10$. We first note that $\mathbf{W}^{8}(4)$ is generated by $x_{1} x_{2} x_{3}^{3} x_{4}^{3}$ and all its permutation representatives, as well as the monomials in the following sets:

$A=\left\{x_{1} x_{2} x_{3}^{2} x_{4}^{4}, x_{1} x_{2}^{2} x_{3} x_{4}^{4}, x_{1} x_{2}^{2} x_{3}^{4} x_{4}\right\}$ and $B=\left\{x_{1}^{3} x_{2} x_{3}^{2} x_{4}^{2}, x_{1} x_{2}^{3} x_{3}^{2} x_{4}^{2}, x_{1} x_{2}^{2} x_{3}^{3} x_{4}^{2}, x_{1} x_{2}^{2} x_{3}^{2} x_{4}^{3}\right\}$.

It is easy to show that the monomial $e_{n-4}$ and all its permutation representatives are inadmissible for all $n \geq 8$.

If $n>8$, then we know that all the permutation representatives of $c_{n-4}$ are admissible. The only permutation representatives of $a_{n-4}$ and $b_{n-4}$ in $\mathbf{X}^{n}(n-4)$ that may not be obtained from the basis of $\mathbf{W}^{8}(4)$ by inductively applying Equation (3) are those of the form $x_{1}^{2} \cdots x_{n-4}^{m_{n-4}}$ and of the form $x_{1}^{3} x_{2}^{2} x_{3}^{m_{3}} x_{4}^{m_{4}} \cdots x_{n-4}^{m_{n-4}}$ as well as those with a factor of the form $x_{i}^{4} x_{j}^{2}, i<j$, all of which are clearly inadmissible. If $n=10$, then by Theorem 7, the following permutation representatives of $d_{6}$ in $\mathbf{X}^{10}(6)$ are admissible, namely those in the set:

$C=\left\{x_{1} x_{2} x_{3}^{2} x_{4}^{2} x_{5}^{2} x_{6}^{2}, x_{1} x_{2}^{2} x_{3} x_{4}^{2} x_{5}^{2} x_{6}^{2}, x_{1} x_{2}^{2} x_{3}^{2} x_{4} x_{5}^{2} x_{6}^{2}, x_{1} x_{2}^{2} x_{3}^{2} x_{4}^{2} x_{5} x_{6}^{2}\right\}$,

The monomial $x_{1} x_{2}^{2} x_{3}^{2} x_{4}^{2} x_{5}^{2} x_{6}$ is inadmissible since

$x_{1} x_{2}^{2} x_{3}^{2} x_{4}^{2} x_{5}^{2} x_{6}=S q^{4}\left(x_{1} x_{2} x_{3} x_{4} x_{5} x_{6}\right)+S q^{3}\left(x_{1}^{2} x_{2} x_{3} x_{4} x_{5} x_{6}\right)+S q^{1}\left(x_{1}^{4} x_{2} x_{3} x_{4} x_{5} x_{6}\right)+x_{1} x_{2}^{2} x_{3}^{2} x_{4}^{2} x_{5} x_{6}^{2}+x_{1} x_{2}^{2} x_{3}^{2} x_{4} x_{5}^{2} x_{6}^{2}$

$+x_{1} x_{2}^{2} x_{3} x_{4}^{2} x_{5}^{2} x_{6}^{2}+x_{1} x_{2} x_{3}^{2} x_{4}^{2} x_{5}^{2} x_{6}^{2}$.

The other permutation representatives of $d_{6}$ are inadmissible since they are of the form $x_{1}^{2} \cdots x_{6}^{m_{6}}$.

If $n>10$, then the only permutation representatives of $d_{n-4}$ in $\mathbf{X}^{n}(n-4)$ that may not be obtained from $d_{6}$ by inductively applying Equation (3) are those of the form $x_{1}^{2} \cdots x_{n-4}^{m_{n-4}}$ as well the monomial $x_{1} x_{2}^{2} x_{3}^{2} x_{4}^{2} x_{5}^{2} x_{6} \cdots x_{n-4}$, all of which are clearly is inadmissible. Thus

$$
\operatorname{dim}\left(\mathbf{W}^{n}(n-4)\right)=\left(\left(\begin{array}{c}
n-5 \\
4
\end{array}\right)-1\right)+\left(\begin{array}{c}
n-4 \\
2
\end{array}\right)+(n-4) \cdot\left(\begin{array}{c}
n-6 \\
2
\end{array}\right)+\left(\begin{array}{c}
n-5 \\
2
\end{array}\right), \text { for all } n \geq 10,
$$

since $a_{n-4}, b_{n-4}, c_{n-4}, d_{n-4}$, have, respectively,

$$
(n-4) \cdot\left(\begin{array}{c}
n-6 \\
2
\end{array}\right),\left(\begin{array}{c}
n-5 \\
2
\end{array}\right),\left(\begin{array}{c}
n-4 \\
2
\end{array}\right),\left(\left(\begin{array}{c}
n-5 \\
4
\end{array}\right)-1\right)
$$

permutation representatives which are admissible. If , $8 \leq n<10$, then we omit the term $\left(\left(\begin{array}{c}n-5 \\ 4\end{array}\right)-1\right)$ in the expression for the value of $\operatorname{dim}\left(\mathbf{W}^{n}(n-4)\right)$.

If $i=5$, then $n_{0}=10$. We first note that $\mathbf{W}^{9}(4)$ is generated by monomials in the following sets:

$A=\left\{x_{1} x_{2} x_{3} x_{4}^{6}, x_{1} x_{2} x_{3}^{6} x_{4}, x_{1} x_{2}^{6} x_{3} x_{4}\right\}, \quad B=\left\{x_{1} x_{2} x_{3}^{2} x_{4}^{5}, x_{1} x_{2}^{2} x_{3} x_{4}^{5}, x_{1} x_{2}^{2} x_{3}^{5} x_{4}\right\}$,

$C=\left\{x_{1} x_{2}^{2} x_{3}^{3} x_{4}^{3}, x_{1} x_{2}^{3} x_{3}^{2} x_{4}^{3}, x_{1} x_{2}^{3} x_{3}^{3} x_{4}^{2}, x_{1}^{3} x_{2} x_{3}^{2} x_{4}^{3}, x_{1}^{3} x_{2} x_{3}^{3} x_{4}^{2}, x_{1}^{3} x_{2}^{3} x_{3} x_{4}^{2}\right\}$, and

$D=\left\{x_{1} x_{2} x_{3}^{3} x_{4}^{4}, x_{1} x_{2}^{3} x_{3} x_{4}^{4}, x_{1} x_{2}^{3} x_{3}^{4} x_{4}, x_{1}^{3} x_{2} x_{3} x_{4}^{4}, x_{1}^{3} x_{2} x_{3}^{4} x_{4}, x_{1}^{3} x_{2}^{4} x_{3} x_{4}\right\}$.

If $n>9$, then the only permutation representative of $a_{n-5}$ in $\mathbf{X}^{n}(n-5)$ that may not be obtained from the basis of $\mathbf{W}^{9}(4)$ by inductively applying Equation (3) is that of the form $x_{1}^{6} x_{2} \cdots x_{n-5}$ which is clearly inadmissible while the only permutation representatives of $b_{n-5}, c_{n-5}$ and $d_{n-5}$ in $\mathbf{X}^{n}(n-5)$ that may not be obtained from the basis of $\mathbf{W}^{9}(4)$ by inductively applying Equation (3) are, respectively, those with a factor of the form $x_{i}^{5} x_{j}^{2}, i<j, x_{i}^{2} x_{j}, i<j$, and $x_{i}^{4} x_{j}^{3}, i<j$, all of which are clearly inadmissible.

If $i=5$ and $n=10$, then by Theorem 7, the following permutation representatives of $e_{5}$ and $f_{5}$ are admissible in $\mathbf{X}^{10}(5)$, namely those in the sets: 
$E=\left\{x_{1}^{3} x_{2} x_{3}^{2} x_{4}^{2} x_{5}^{2}, x_{1} x_{2}^{3} x_{3}^{2} x_{4}^{2} x_{5}^{2}, x_{1} x_{2}^{2} x_{3}^{3} x_{4}^{2} x_{5}^{2}, x_{1} x_{2}^{2} x_{3}^{2} x_{4}^{3} x_{5}^{2}, x_{1} x_{2}^{2} x_{3}^{2} x_{4}^{2} x_{5}^{3}\right\}$,

$F=\left\{x_{1} x_{2} x_{3}^{2} x_{4}^{2} x_{5}^{4}, x_{1} x_{2} x_{3}^{2} x_{4}^{4} x_{5}^{2}, x_{1} x_{2}^{2} x_{3} x_{4}^{2} x_{5}^{4}, x_{1} x_{2}^{2} x_{3}^{4} x_{4} x_{5}^{2}, x_{1} x_{2}^{2} x_{3} x_{4}^{4} x_{5}^{2}\right\}$.

Permutation representatives of $e_{5}$ which are not in $E$ are those of the form $x_{1}^{2} \cdots x_{5}^{m_{5}}$ as well as those of the form $x_{1}^{3} x_{2}^{2} x_{3}^{m_{3}} \cdots x_{5}^{m_{5}}$, all of which are clearly inadmissible. Permutation representatives of $f_{5}$ which are not in $F$ are those of the form $x_{1}^{2} \cdots x_{5}^{m_{5}}$ as well as those with a factor of the form $x_{i}^{4} x_{j}^{2} x_{k}^{2}, i<j, k$, and the three monomials in the set $G=\left\{x_{1} x_{2}^{2} x_{3}^{4} x_{4}^{2} x_{5}, x_{1} x_{2}^{2} x_{3}^{2} x_{4}^{4} x_{5}, x_{1} x_{2}^{2} x_{3}^{2} x_{4} x_{5}^{4}\right\}$, all of which are clearly inadmissible. For example

$$
x_{1} x_{2}^{2} x_{3}^{4} x_{4}^{2} x_{5}=S q^{2}\left(x_{1} x_{2} x_{3}^{4} x_{4} x_{5}\right)+S q^{1}\left(x_{1}^{2} x_{2} x_{3}^{4} x_{4} x_{5}\right)+x_{1} x_{2}^{2} x_{3}^{4} x_{4} x_{5}^{2}+x_{1} x_{2} x_{3}^{4} x_{4}^{2} x_{5}^{2} .
$$

If $n>10$, then the only permutation representatives of $e_{n-5}$ in $\mathbf{X}^{n}(n-5)$ that may not be obtained from the basis of $\mathbf{W}^{10}(5)$ by inductively applying Equation (3) are those of the form $x_{1}^{2} \cdots x_{n-5}^{m_{n-5}}$ as well as those of the form $x_{1}^{3} x_{2}^{2} x_{3}^{m_{3}} \cdots x_{n-5}^{m_{n-5}}$, all of which are clearly inadmissible. On the other hand the permutation representatives of $f_{n-5}$ that may not be obtained from the basis of $\mathbf{W}^{10}(5)$ by inductively applying Equation (3) are those of the form $x_{1}^{2} \cdots x_{n-5}^{m_{n-5}}$ as well as those with a factor of the form $x_{i}^{4} x_{j}^{2} x_{k}^{2}, i<j, k$, and the three monomials in the set:

$$
G=\left\{x_{1} x_{2}^{2} x_{3}^{4} x_{4}^{2} x_{5} \cdots x_{n-5}, x_{1} x_{2}^{2} x_{3}^{2} x_{4}^{4} x_{5} \cdots x_{n-5}, x_{1} x_{2}^{2} x_{3}^{2} x_{4} x_{5}^{4} \cdots x_{n-5}\right\}
$$

all of which are clearly inadmissible. Thus

$$
\operatorname{dim}\left(\mathbf{W}^{n}(n-5)\right) \geq n-6+\frac{(n-5) !}{2 \cdot(n-7)(n-9) !}+\left(\begin{array}{c}
n-5 \\
2
\end{array}\right)+\left(\begin{array}{c}
n-6 \\
2
\end{array}\right)+(n-5) \cdot\left(\begin{array}{c}
n-7 \\
3
\end{array}\right)+2 \cdot\left(\sum_{i=2}^{n-8}\left(\begin{array}{c}
i \\
2
\end{array}\right)\right)+\left(\begin{array}{c}
n-8 \\
2
\end{array}\right)+\left(\left(\begin{array}{c}
n-7 \\
2
\end{array}\right)-1\right),
$$

since $a_{n-5}, b_{n-5}, c_{n-5}, d_{n-5}, e_{n-5}, f_{n-5}$, have, respectively,

$$
\frac{(n-5) !}{2 \cdot(n-7)(n-9) !},\left(\begin{array}{c}
n-5 \\
2
\end{array}\right),\left(\begin{array}{c}
n-6 \\
2
\end{array}\right), n-6,(n-5) \cdot\left(\begin{array}{c}
n-7 \\
3
\end{array}\right), 2 \cdot\left(\sum_{i=2}^{n-8}\left(\begin{array}{c}
i \\
2
\end{array}\right)\right)+\left(\begin{array}{c}
n-8 \\
2
\end{array}\right)+\left(\left(\begin{array}{c}
n-7 \\
2
\end{array}\right)-1\right),
$$

permutation representatives which are admissible. Equality holds when, $10 \leq n \leq 12$.

If $i=6$ then $n_{0}=11$. We know that all permutation representatives of $a_{n-6}$ and $b_{n-6}$ are admissible for all $n \geq 11$. By Theorem 7, the following permutation representatives of $c_{5}, d_{5}, e_{5}, f_{5}$ and $g_{5}$ are admissible in $\mathbf{X}^{11}(5)$, namely those in the sets:

$A=\left\{x_{1} x_{2}^{2} x_{3}^{2} x_{4}^{3} x_{5}^{3}, x_{1} x_{2}^{2} x_{3}^{3} x_{4}^{2} x_{5}^{3}, x_{1} x_{2}^{3} x_{3}^{2} x_{4}^{2} x_{5}^{3}, x_{1}^{3} x_{2} x_{3}^{2} x_{4}^{2} x_{5}^{3}, x_{1} x_{2}^{2} x_{3}^{3} x_{4}^{3} x_{5}^{2}, x_{1} x_{2}^{3} x_{3}^{2} x_{4}^{3} x_{5}^{2}, x_{1}^{3} x_{2} x_{3}^{2} x_{4}^{3} x_{5}^{2}, x_{1} x_{2}^{3} x_{3}^{3} x_{4}^{2} x_{5}^{2}, x_{1}^{3} x_{2} x_{3}^{3} x_{4}^{2} x_{5}^{2}\right.$, $\left.x_{1}^{3} x_{2}^{3} x_{3} x_{4}^{2} x_{5}^{2}\right\}$,

$B=\left\{x_{1} x_{2}^{2} x_{3} x_{4}^{6} x_{5}, x_{1} x_{2}^{2} x_{3} x_{4} x_{5}^{6}, x_{1} x_{2} x_{3}^{2} x_{4} x_{5}^{6}, x_{1} x_{2} x_{3} x_{4}^{2} x_{5}^{6}, x_{1} x_{2} x_{3} x_{4}^{6} x_{5}^{2}, x_{1} x_{2}^{6} x_{3} x_{4}^{2} x_{5}, x_{1} x_{2}^{6} x_{3} x_{4} x_{5}^{2}, x_{1} x_{2} x_{3}^{6} x_{4}^{2} x_{5}, x_{1} x_{2} x_{3}^{6} x_{4} x_{5}^{2}\right.$, $\left.x_{1} x_{2} x_{3}^{2} x_{4}^{6} x_{5}\right\}$,

$C=\left\{x_{1} x_{2}^{3} x_{3}^{4} x_{4} x_{5}^{2}, x_{1} x_{2}^{3} x_{3}^{4} x_{4}^{2} x_{5}, x_{1} x_{2}^{3} x_{3} x_{4}^{4} x_{5}^{2}, x_{1} x_{2} x_{3}^{3} x_{4}^{4} x_{5}^{2}, x_{1}^{3} x_{2} x_{3}^{4} x_{4} x_{5}^{2}, x_{1}^{3} x_{2} x_{3} x_{4}^{4} x_{5}^{2}, x_{1}^{3} x_{2}^{4} x_{3} x_{4}^{2} x_{5}, x_{1}^{3} x_{2}^{4} x_{3} x_{4} x_{5}^{2}, x_{1}^{3} x_{2} x_{3}^{4} x_{4}^{2} x_{5}\right.$, $x_{1}^{3} x_{2} x_{3} x_{4}^{2} x_{5}^{4}, x_{1} x_{2}^{3} x_{3} x_{4}^{2} x_{5}^{4}, x_{1} x_{2} x_{3}^{3} x_{4}^{2} x_{5}^{4}, x_{1} x_{2} x_{3}^{2} x_{4}^{3} x_{5}^{4}, x_{1} x_{2} x_{3}^{2} x_{4}^{4} x_{5}^{3}, x_{1}^{3} x_{2} x_{3}^{2} x_{4} x_{5}^{4}, x_{1} x_{2}^{3} x_{3}^{2} x_{4} x_{5}^{4}, x_{1} x_{2}^{2} x_{3}^{3} x_{4} x_{5}^{4}, x_{1} x_{2}^{2} x_{3} x_{4}^{3} x_{5}^{4}$,

$\left.x_{1} x_{2}^{2} x_{3} x_{4}^{4} x_{5}^{3}, x_{1}^{3} x_{2} x_{3}^{2} x_{4}^{4} x_{5}, x_{1} x_{2}^{3} x_{3}^{2} x_{4}^{4} x_{5}, x_{1} x_{2}^{2} x_{3}^{3} x_{4}^{4} x_{5}, x_{1} x_{2}^{2} x_{3}^{4} x_{4}^{3} x_{5}, x_{1} x_{2}^{2} x_{3}^{4} x_{4} x_{5}^{3}\right\}$,

$D=\left\{x_{1} x_{2} x_{3} x_{4}^{3} x_{5}^{5}, x_{1} x_{2} x_{3}^{3} x_{4} x_{5}^{5}, x_{1} x_{2}^{3} x_{3} x_{4} x_{5}^{5}, x_{1}^{3} x_{2} x_{3} x_{4} x_{5}^{5}, x_{1} x_{2} x_{3}^{3} x_{4}^{5} x_{5}, x_{1} x_{2}^{3} x_{3} x_{4}^{5} x_{5}, x_{1}^{3} x_{2} x_{3} x_{4}^{5} x_{5}, x_{1} x_{2}^{3} x_{3}^{5} x_{4} x_{5}, x_{1}^{3} x_{2} x_{3}^{5} x_{4} x_{5}\right.$, $\left.x_{1}^{3} x_{2}^{5} x_{3} x_{4} x_{5}\right\}$

$E=\left\{x_{1} x_{2} x_{3}^{2} x_{4}^{5} x_{5}^{2}, x_{1} x_{2}^{2} x_{3} x_{4}^{5} x_{5}^{2}, x_{1} x_{2}^{2} x_{3}^{5} x_{4} x_{5}^{2}, x_{1} x_{2}^{2} x_{3}^{5} x_{4}^{2} x_{5}, x_{1} x_{2}^{2} x_{3} x_{4}^{2} x_{5}^{5}, x_{1} x_{2} x_{3}^{2} x_{4}^{2} x_{5}^{5}\right\}$.

It is known, (Mothebe 2009), that $\operatorname{dim}\left(\mathbf{Q}^{11}(5)\right)=315$, so the above permutation representatives of the monomials of $a_{5}, b_{5} c_{5}, d_{5}, e_{5}, f_{5}$ and $g_{5}$ form a complete list of admissible monomials in $\mathbf{X}^{11}(5)$. We claim that

$$
\mathbf{W}^{12}(6)=\sum_{j=1}^{6} h_{j}^{1}\left(\mathbf{W}^{11}(5)\right) \text {. }
$$

If $n>11$, then the only permutation representatives of $c_{n-6}, d_{n-6}, e_{n-6}, f_{n-6}$ and $g_{n-6}$ in $\mathbf{X}^{n}(n-6)$ that may not be obtained from the basis of $\mathbf{W}^{11}(5)$ by inductively applying Equation (3) are those of the form $x_{1}^{2} \cdots x_{n-6}^{m_{n-6}}, x_{1}^{3} x_{2}^{2} \cdots x_{n-6}^{m_{n-6}}$ or, respectively, those of the form $x_{1}^{3} x_{2}^{3} x_{3}^{2} \cdots x_{n-6}^{m_{n-6}}, x_{1}^{6} \cdots x_{n-6}^{m_{n-6}}$ or $x_{1} x_{2}^{2} x_{3}^{6} x_{4} \cdots x_{n-6}$ or $x_{1} x_{2}^{6} x_{3}^{2} x_{4} \cdots x_{n-6}, x_{1}^{4} \cdots x_{n-6}^{m_{n-6}}$ or $x_{1}^{3} x_{2}^{4} x_{3}^{2} \cdots x_{n-6}$ or $x_{1} x_{2}^{4} \cdots x_{n-6}^{m_{n-6}}$ or $x_{1} x_{2} x_{3}^{4} \cdots x_{n-6}^{m_{n-6}}$, those with a factors of the form $x_{i}^{5} x_{j}^{3}, i<j, x_{1} x_{2}^{2} x_{3}^{2} \cdots x_{n-6}^{m_{n-6}}$ or those with a factors of the form $x_{i}^{5} x_{j}^{2} x_{k}^{2}, i<j, k$, all of which are clearly inadmissible. Thus

$$
\begin{aligned}
\operatorname{dim}\left(\mathbf{W}^{n}(n-6)\right) \geq & n-6+((n-7)(n-8)-2)+\left(\begin{array}{c}
n-6 \\
2
\end{array}\right)+\left(\begin{array}{c}
n-6 \\
3
\end{array}\right)+2 \cdot\left(\begin{array}{c}
n-7 \\
3
\end{array}\right)-(n-9) \\
& +(n-6) \cdot\left(\begin{array}{c}
n-8 \\
2
\end{array}\right)+\left(\begin{array}{c}
n-6 \\
3
\end{array}\right)-1+\frac{(n-7) !}{4 \cdot(n-11) !}+(n-8) \cdot\left(\begin{array}{c}
n-9 \\
2
\end{array}\right)+\left(\begin{array}{c}
n-9 \\
2
\end{array}\right),
\end{aligned}
$$


since $a_{n-6}, b_{n-6}, c_{n-6}, d_{n-6}, e_{n-6}, f_{n-6}, g_{n-6}$, have, respectively,

$\left(\begin{array}{c}n-6 \\ 3\end{array}\right), n-6,\left(\begin{array}{c}n-6 \\ 2\end{array}\right), \frac{(n-7) !}{4 \cdot(n-11) !}+(n-8) \cdot\left(\begin{array}{c}n-9 \\ 2\end{array}\right)+\left(\begin{array}{c}n-9 \\ 2\end{array}\right),(n-6) \cdot\left(\begin{array}{c}n-8 \\ 2\end{array}\right)+\left(\begin{array}{c}n-6 \\ 3\end{array}\right)-1,(n-7)(n-8)-2,2 \cdot\left(\begin{array}{c}n-7 \\ 3\end{array}\right)-(n-9)$

permutation representatives which are admissible. Equality holds when $n=11,12$.

It is known, (Sum \& Phuc, 2013), that $\operatorname{dim}\left(\mathbf{Q}^{12}(5)\right)=190$. Since $\operatorname{dim}\left(\mathbf{Q}^{12}(4)\right)=21$, we must have $\operatorname{dim}\left(\mathbf{W}^{12}(5)\right)=85$. This completes the proof of the lemma hence that of Theorem 2.

\section{References}

Adem, J. (1957). The relations on Steenrod powers of cohomology classes. Algebraic Geometry and Topology, a symposium in honour of S. Lefschetz, Princeton Univ. Press, Princeton NJ, 191-238.

Kameko, M. (1990). Products of projective spaces as Steenrod modules. Ph.D. Thesis, John Hopkins University, USA.

Kameko, M. (2003). Generators of the cohomology of $B V_{4}$ Preprint: Toyama Univ.

Mothebe, M. F. (2016). Products of admissible monomials in the polynomial algebra as a module over the Steenrod algebra. Journal of Mathematics Research, 8(3), 112-116. http://dx.doi.org/10.5539/jmr.v8n3p112

Mothebe, M. F., \& Uys, L. (2015). Some Relations between admissible monomials for the polynomial algebr. International Journal of Mathematics and Mathematical Sciences. http://dx.doi.org/10.1155/2015/235806

Mothebe, M. F. (2009). Dimensions of the polynomial algebra $\mathbb{F}_{2}\left[x_{1}, \ldots, x_{n}\right]$ as a module over the Steenrod algebra. JP Journal of Algebra, Number Theory and its Applications, 13(2), 161-170.

Nam, T. N. (2004). A-générateurs génériques pour l' algèbre polynomiale. Advances in Mathematics, 186, $334-362$. http://dx.doi.org/10.1016/j.aim.2003.08.004

Peterson, F. P. (1987). Generators of $H^{*}\left(R P^{\infty} \wedge R P^{\infty}\right)$ as a module over the Steenrod algebra. Abstracts American Mathematical Society, 833, 55-89.

Peterson, F. P. (1989). A-generators for certain polynomial algebras. Mathematical Proceedings of the Cambridge Philosophical Society, 105, 311-312. http://dx.doi.org/10.1017/S0305004100067803

Silverman, J. H. (1998). Hit polynomials and conjugation in the dual Steenrod algebra Mathematical Proceedings of the Cambridge Philosophical Societys, 123, 531-547. http://dx.doi.org/10.1017/S0305004197002302

Singer, W. M. (1989). The transfer in homological algebra. Mathematische Zeitschrift, 202, 493-523. http://dx.doi.org/10.1007/BF01221587

Singer, W. M. (1991). On the action of Steenrod squares on polynomials. Proceedings of the American Mathematical Society, 111, 577-583. http://dx.doi.org/ 10.2307/2048351

Sum, N. (2007). The hit problem for the polynomial algebra of four variables. Preprint, University of Quy Nhon, Viet Nam.

Sum, N. (2010). The negative answer to Kameko's conjecture on the hit problem. Advances in Mathematics, 225, 23652390.

Sum, N., \& Phuc, D. V. (2013). On a minimal set of generators for the polynomial algebra of five variables as a module over the Steenrod algebra. Preprint, University of Quy Nhon, Viet Nam.

Wood, R. M. W. (1989). Steenrod squares of polynomials and the Peterson conjecture. Mathematical Proceedings of the Cambridge Philosophical Societys, 105, 307-309. http://dx.doi.org/10.1017/S0305004100067797

\section{Copyrights}

Copyright for this article is retained by the author(s), with first publication rights granted to the journal.

This is an open-access article distributed under the terms and conditions of the Creative Commons Attribution license (http://creativecommons.org/licenses/by/4.0/). 